\title{
Direction-of-motion discrimination is facilitated by visible motion smear
}

\author{
Jianliang Tong, Murat Aydin, and Harold E. Bedell \\ University of Houston, Houston, Texas
}

\begin{abstract}
Recent evidence indicates that motion smear can provide useful information for the detection and discrimination of motion. Further, it has been shown that the perception of motion smear depends critically on the density of dots in a random-dot (RD) stimulus. Therefore, in the present experiments, the contribution of perceived motion smear to direction-of-motion discrimination was evaluated using RD targets of different densities. Thresholds for direction-of-motion discrimination and the extent of perceived motion smear were determined for RD stimuli with densities of 1,2 , and $10 \mathrm{dots} / \mathrm{deg}^{2}$, presented for $200 \mathrm{msec}$ at a velocity of 4,8 , or $12 \mathrm{deg} / \mathrm{sec}$. To evaluate the contribution of information about orientation from motion smear, thresholds for orientation discrimination were measured using parallel lines with the same length as the extent of perceived smear. Despite the opportunity for increased summation as RD density increases, our results indicate that direction-of-motion discrimination worsens. Because perception of motion smear is reduced with an increase in RD density, our results are consistent with a facilitation of direction-of-motion discrimination by visible motion smear.
\end{abstract}

One of the fundamental tasks of the visual system is to decode the direction information in the retinal-image motion that results from objects that move in space. Electrophysiological investigations have disclosed that nerve cells located in striate and extrastriate cortical areas have direction-selective characteristics (Cheng, Fujita, Kanno, Miura, \& Tanaka, 1995; Cornette et al., 1998; Hubel \& Wiesel, 1968; Singh, Smith, \& Greenlee, 2000), and it is generally assumed that the brain constructs its percept of the direction of motion from the selective responses of such neurons (Azzopardi \& Cowey, 2001; Blanke, Landis, Mermoud, Spinelli, \& Safran, 2003; Britten, Shadlen, Newsome, \& Movshon, 1992; Salzman, Britten, \& Newsome, 1990). However, debate continues as to how these cortical neurons work together to generate the perception of motion (Adelson \& Movshon, 1982; Marr \& Ullman, 1981; Pack, Livingstone, Duffy, \& Born, 2003; Peterson, Li, \& Freeman, 2004; Purushothaman \& Bradley, 2005; D. W. Williams \& Sekuler, 1984; Zohary, Scase, \& Braddick, 1996).

Geisler (1999) hypothesized that spatial orientation information from "motion streaks" may be used by the visual system to enhance the encoding of moving targets. The visual representation of an object that moves at a sufficient velocity should be smeared, because of the visual persistence that accompanies temporal integration (Bidwell, 1899; Bowen, Pola, \& Matin, 1974; Coltheart, 1980; McDougall, 1904). This motion smear produces a streak in the orientation parallel to the direction of motion. Neurons in the primary visual cortex tuned to orientations parallel to the motion trajectory should be activated by the streak, and the output from these orientation-selective detectors could combine with that from direction-selective detectors to determine the direction of motion. Geisler, Albrecht, Crane, and Stern (2001) presented neurophysiological evidence to indicate that orientation-tuned neurons in the primary visual cortex of cat and monkey do, in fact, respond to motion streaks. A related proposal by Barlow and Olshausen (2004) suggests that the visual system uses the anisotropies of local spatial frequency power spectra that result from motion blur to analyze the direction of motion streaks.

Support for a contribution of motion streaks to the processing of visual motion is available from psychophysical experiments. Geisler (1999) reported that adaptation to a tilted grating significantly shifts the perceived direction of a bright spot that moves vertically at $10 \mathrm{deg} / \mathrm{sec}$, but does not do the same for a spot that moves at $2.5 \mathrm{deg} / \mathrm{sec}$. In this experiment, the moving bright spot was presented on a dark background, which, when the velocity was $10 \mathrm{deg} / \mathrm{sec}$, would be expected to produce a noticeable extent of visible motion smear. Subsequently, Burr and Ross (2002) showed that the discrimination of direction for limited-lifetime, moving random-dot (RD) stimuli is impaired preferentially by masking stimuli that are oriented parallel to the direction of motion. Because the RD stimuli in this experiment were of low density $\left(<1 \mathrm{dot} / \mathrm{deg}^{2}\right)$ and moved at $32 \mathrm{deg} / \mathrm{sec}$, they should have provided substantial orientation information.

The possible relationship between neural mechanisms sensitive to orientation and motion was also addressed by comparing the thresholds for motion direction with those for line orientation, using oriented targets with a length that was equal to the motion trajectory (Westheimer \& Wehrhahn, 1994). The results of this study suggest that 
the discrimination of orientation and direction of motion may share the same neural mechanisms. However, a recent study by Matthews and Allen (2005) called the contribution of motion streaks into question. This study found that direction discrimination is poorer for pairs of stimuli that move in the opposite direction than for those that move in the same direction, even though both types of stimuli should provide the same orientation information. This observation is considered in more detail in the Discussion section.

In the present study, we addressed the relationship between direction-of-motion discrimination and the extent of perceived motion smear. RD stimuli with different densities were used to manipulate the extent of perceived motion smear, at each of several retinal-image velocities (Chen, Bedell, \& Ögmen, 1995; Purushothaman, Ögmen, Chen, $\&$ Bedell, 1998). The angular thresholds to discriminate the direction of RD motion were compared with the thresholds to discriminate the orientation of briefly presented lines that were equal in length to the perceived motion smear, to evaluate the contribution of orientation information to direction-of-motion discrimination.

\section{METHOD}

Visual stimuli were generated by a PC-controlled 12-bit arbitrary waveform generator and presented on an $\mathrm{X}-\mathrm{Y}$ monitor (HP 1311B) at a 500-Hz refresh rate. Stimuli were presented at a luminance of $2 \log$ units (approximately $14 \mathrm{~cd} / \mathrm{m}^{2}$ ) above the detection threshold in an otherwise dark environment and were viewed monocularly with the left eye through a mirror system. To measure direction-of-motion discrimination and the extent of perceived motion smear, we presented bright RDs on a dark background with a dot size of 0.4 arc min and densities of 1, 2, or $10 \mathrm{dots} / \mathrm{deg}^{2}$. The dots $(n=1,2$, or 10$)$ were distributed randomly within a $1 \mathrm{deg} \times 1 \mathrm{deg}$ virtual square on the screen. The spatial separation between elements was at least $0.5 \mathrm{deg}$ when the density was $2 \mathrm{dots} / \mathrm{deg}^{2}$, and at least $0.1 \mathrm{deg}$ when the density was 10 dots $/ \mathrm{deg}^{2}$. All of the dots moved with the same speed and direction on each trial, and the trajectories of the individual dots did not overlap. To measure orientation discrimination, we presented between 1 and 10 stationary parallel lines, with the midpoint of each line distributed randomly within a $1 \mathrm{deg} \times 1 \mathrm{deg}$ virtual square. At the subject's viewing distance of $4 \mathrm{~m}$, the entire screen subtended an angle of 4 deg horizontally $\times 3$ deg vertically. An 8.5 arc min LED, reflected to the left eye by a beam splitter, served as the fixation target (see Figure 1).

The fixation target was extinguished during each presentation of the moving $\mathrm{RD}$ or stationary line stimulus to eliminate possible reference information about the direction of motion or orientation. Horizontal motion of the RDs was generated by rotating a galvanometermounted mirror, from which the observer viewed the entire stimulus display. Only the small upward or downward component of RD motion on each trial was produced by physical motion of the dots across the screen. This strategy minimized the possibility that the motion of the dots would generate visible screen persistence. Further, a synchronized shutter eliminated the perception of residual screen persistence after each stimulus presentation. The duration of each stimulus presentation was $200 \mathrm{msec}$, to reduce the influence of pursuit eye movements on motion discrimination. Nevertheless, it has been shown that

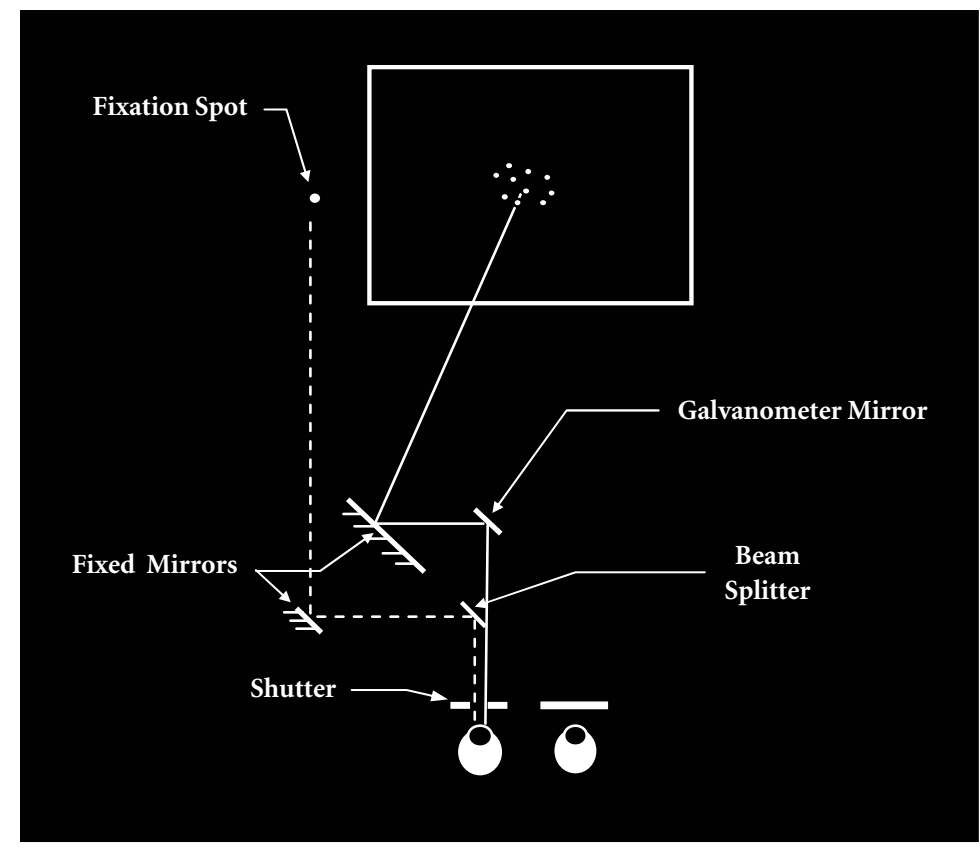

Figure 1. Schematic diagram of the experimental setup. An 8.5 arc min LED that served as the fixation target was reflected to the left eye of the subject by a fixed mirror and a fixed beam splitter. A patch covered the subject's right eye. The horizontal component of stimulus motion was achieved by rotating a galvanometer-mounted mirror. A computer-controlled shutter in front of the left eye was synchronized to remain open concurrently with the presentation of the stimulus. 

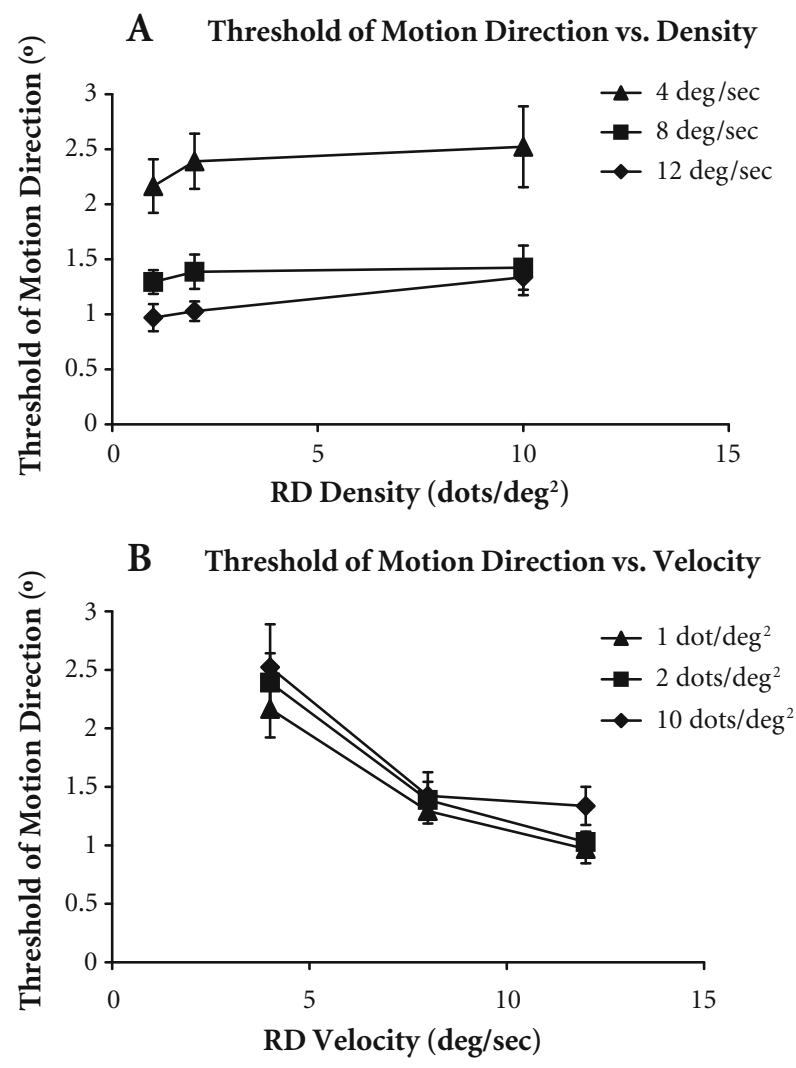

Figure 2. The threshold for direction discrimination is shown as a function of RD density (panel $A$ ) and $R D$ velocity (panel B). (A) The threshold to discriminate the direction of motion increases as the stimulus density increases. For each RD density, the threshold increases with a decrease of stimulus velocity. (B) The threshold to discriminate the direction of motion decreases as the stimulus velocity increases. For each velocity, the threshold decreases as the stimulus density is reduced. Each data point represents the average of 6 subjects $\pm 1 S E$.

the discrimination of the direction of motion is not based on the direction of tracking eye-movement responses (Holtzman, Sedgwick, \& Festinger, 1978; Kosnik, Fikre, \& Sekuler, 1985).

\section{Experiment 1: Direction Discrimination and Extent} of Perceived Motion Smear for Moving Random Dots

On each presentation, an RD stimulus with one of three densities $\left(1,2\right.$, or $\left.10 \mathrm{dots} / \mathrm{deg}^{2}\right)$ moved at one of three rightward velocities: 4,8 , or $12 \mathrm{deg} / \mathrm{sec}$. At these velocities, the trajectory of motion extended $0.8,1.6$, or $2.4 \mathrm{deg}$ for each dot, respectively. For each combination of dot density and velocity, the direction-of-motion threshold was determined by presenting the dots in near-horizontal directions of motion. The horizontal component of RD-stimulus motion was always in the direction of left to right. On each trial, the vertical component of the near-horizontal RD motion was chosen from seven possible values. Appropriate values were determined for each subject individually, in pilot experiments. After each trial, the subject used a joystick to report whether the motion of the RD target included an upward or a downward component. Each individual estimate of the direction-of-motion threshold was determined from a set of 70 trials using the method of constant stimuli.

In separate sets of trials, the same RD targets were presented for $200 \mathrm{msec}$, moving in the left-to-right direction. After each trial, the subject adjusted the length of a bright horizontal line to match the extent of perceived motion smear (see, e.g., Tong, Patel, \& Bedell,
2005). As described previously (Chen et al., 1995), the subjects reported that the moving dots were followed by a continuous bright tail, or by a bright tail and a dimmer tail separated by an intercalated dark region. The subjects were instructed to match the entire length of the perceived smear, including the dark region, if it was present. Estimates of perceived motion smear for each combination of dot density and velocity were averaged across 10 to 20 matches per subject and condition.

\section{Experiment 2: Orientation Discrimination for Flashed \\ Random Lines}

In this experiment, the stimuli consisted of 0.4-arc-min-wide parallel random lines (RLs), with densities of 1,2 , or 10 lines/ $/ \mathrm{deg}^{2}$. The spatial separation between lines was the same as for the dots in Experiment 1. Nine different line lengths were tested for each subject, corresponding to the average extent of perceived motion smear that was determined for the nine combinations of RD density and velocity of motion in Experiment 1 . Each estimate of the orientation-discrimination threshold was determined by presenting lines in seven near-horizontal orientations in a set of 70 trials. The subject reported after each trial whether the right side of the RLs was tilted up or down.

\section{Subjects and Data Analysis}

Six subjects with normal or corrected-to-normal vision participated in the experiments. Head position was maintained using a chinrest. Thresholds were defined as the change in the direction of motion or line orientation that corresponds to $1 S D$ on the psychometric function fit to the data using Probit analysis. The confidence limit for statistical comparisons was set at $95 \%$. The plotted values for each subject are the average of three or more threshold estimates. The experimental protocols were reviewed by the University of Houston Committee for the Protection of Human Subjects. Written, informed consent was obtained from the subjects before they participated.

\section{RESULTS}

\section{Experiment 1}

In the first experiment, we addressed the relationship between the threshold for discriminating the direction of motion and the extent of perceived motion smear, using RD patterns with different densities and velocities of motion. When compared with the luminance of the stimuli in previous motion-detection experiments (Burr \& Ross, 2002; Geisler, 1999), the luminance of the present stimuli was substantially above the detection threshold. The direction-of-motion thresholds averaged across all observers are plotted against the density of RDs in Figure 2A and against the velocity of RD motion in Figure 2B. To distinguish between units of angle and angular distance, we use the degree symbol $\left({ }^{\circ}\right)$ to specify direction-of-motion and orientation thresholds, and "deg" to specify the angular extent of perceived motion smear or line length. Error bars represent $\pm 1 S E$, across subjects. A repeated measures ANOVA indicated that the direction-of-motion threshold increases significantly with RD density $[F(2,10)=4.709$, $p=.048]$ and decreases significantly with RD velocity $[F(2,10)=27.305, p=.001]$.

A pilot experiment indicated that small deviations from horizontal in the direction of dot motion had no significant effect on the extent of perceived motion smear. Consequently, only RD stimuli that moved in the horizontal direction were used to measure the length of perceived 
motion smear. The angular length of perceived smear averaged across all observers is plotted against the density of the moving RDs in Figure 3A and against the velocity of RD motion in Figure 3B. When compared with results for the same stimulus conditions in Figure 2, the trends shown in Figure 3 are clearly in the opposite direction. A repeated measures ANOVA indicated that the angular length of perceived motion smear decreases significantly as the $\mathrm{RD}$ density increases $[F(2,10)=7.634, p=.038]$ and increases significantly as the RD velocity increases $[F(2,10)=15.967, p=.008]$.

\section{Experiment 2}

Our experimental procedure allowed us to compare directly the discrimination thresholds for direction of motion and orientation, using lines that were equal in length to the extent of perceived motion smear. The disadvantage of this procedure was that the individual line lengths differed from subject to subject for the same conditions. For this reason, we did not perform an ANOVA on these data. Figure 4 shows the averaged orientation-discrimination

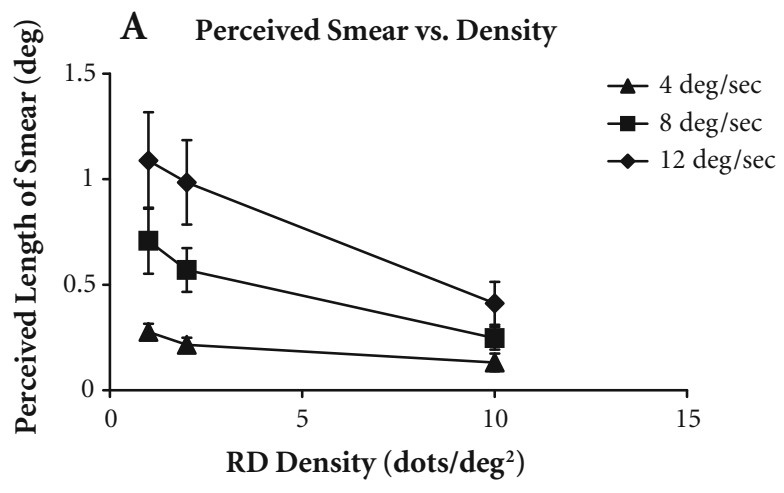

B Perceived Smear vs. Velocity

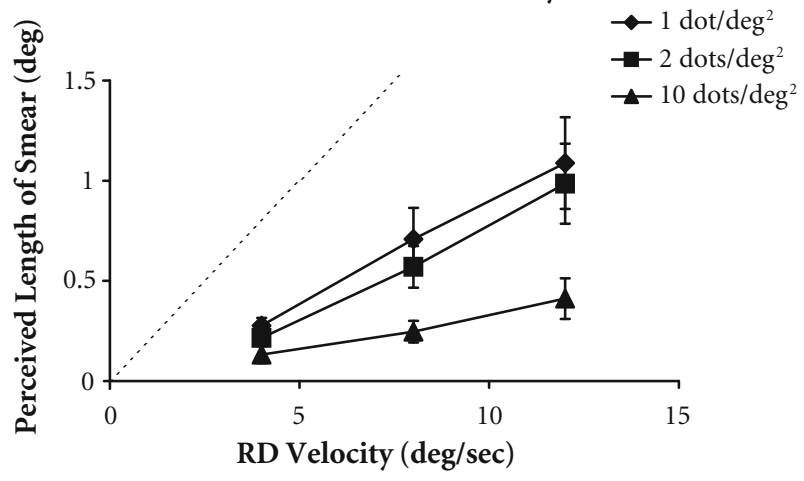

Figure 3. The extent of perceived motion smear is plotted as a function of RD density (panel A) and RD velocity (panel B). The extent of perceived motion smear is expressed in units of degrees. (A) The extent of perceived motion smear decreases as the stimulus density increases. For each density, perceived smear decreases with a decrease of stimulus velocity. (B) The extent of perceived motion smear increases as the stimulus velocity increases. For each velocity, the extent of perceived motion smear increases with a decrease of stimulus density. The dashed line in panel B shows the length of the moving stimulus trajectory for each velocity. Error bars represent $\pm 1 S E$ across subjects.

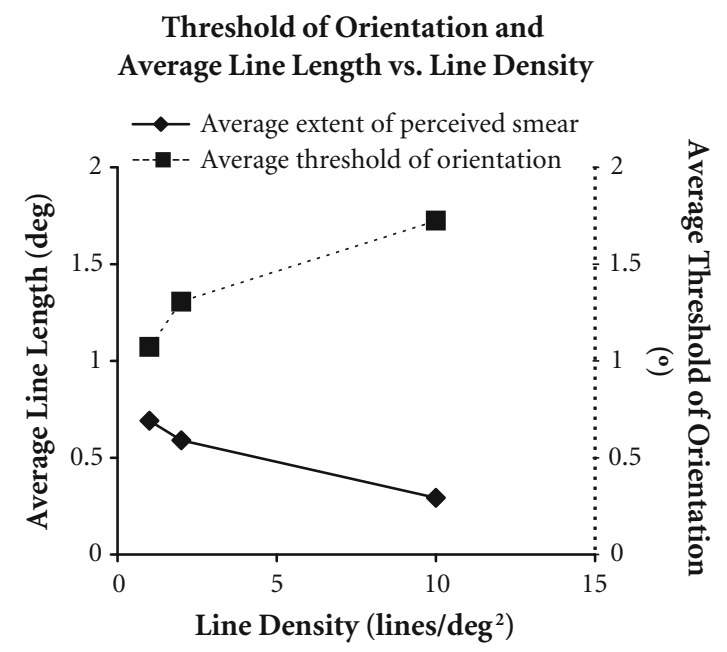

Figure 4. The threshold for orientation discrimination and the corresponding extent of perceived motion smear are plotted as a function of RL density. For orientation discrimination, each data point (square symbols connected by dashed lines) is the average across all subjects and line lengths for each dot density, weighted by the inverse $S E$ s. For perceived motion smear, each data point (diamond-shaped symbols connected by solid lines) is the unweighted average across all subjects and RD velocities for each dot density. The orientation threshold increases and the extent of perceived motion smear decreases as the line density increases.

thresholds plotted on the right-hand ordinate as a function of the line density. Because line length was set equal to the extent of perceived motion smear in each condition, the figure also plots the average line length (left-hand ordinate) used for each density. To calculate the average orientationdiscrimination threshold across subjects for each line density, we weighted each subject's threshold by its inverse $S E$. The figure shows that the orientation-discrimination threshold increases with the density of RLs, as expected from the associated reduction of line length (Ukwade, Bedell, \& White, 2002; Westheimer \& Wehrhahn, 1994; R. A. Williams, Enoch, \& Essock, 1984).

\section{Comparison of Experiments 1 and 2}

Figure 5A plots the ratio of the orientation-discrimination and motion-direction thresholds against the matched values of line length and extent of perceived smear for each subject and experimental condition. The data are described using a "two-line" fit, in which the second line is constrained to have a slope of zero. The $y$-intercept of the second line is 0.99 and the best-fitting lines intersect at an $x$ value of 0.18 , which indicates that orientationdiscrimination and direction-of-motion thresholds are virtually identical when the extent of perceived motion smear is greater than approximately $0.2 \mathrm{deg}$. The plotted ratios clearly increase when the line and smear lengths are less than approximately $0.2 \mathrm{deg}$, indicating that orientation thresholds are systematically higher than directionof-motion thresholds for the stimuli in these conditions.

Figure 5B plots the average direction-of-motion thresholds versus the average line-orientation thresholds for comparable stimulus conditions. Specifically, the average 
direction-of-motion threshold was determined for each combination of RD density and velocity, and the (weighted) average line-orientation threshold was calculated for each combination of RL density and line length. Vertical and horizontal error bars are $\pm 1 S E$, across subjects. The 1:1 line in the figure represents equality between direction-ofmotion discrimination and orientation discrimination. The data points above this line mean that subjects are more sensitive to line orientation than to direction of motion, and vice versa. The plotted points are closest to the diagonal 1:1 line for combinations of high RD velocity and low
RD density. As indicated by the results of Experiment 1, these are the conditions that generate the most appreciable extent of perceived motion smear. On the contrary, the two data points that fall below the 1:1 line are obtained for RD densities of $10 \mathrm{dots} / \mathrm{deg}^{2}$ and velocities of 4 and $8 \mathrm{deg} / \mathrm{sec}$, which produced a reduced extent of perceived smear in comparison with the other conditions. Average thresholds for direction and orientation discrimination were similar for $10 \mathrm{dots} / \mathrm{deg}^{2}$ stimuli that moved at $12 \mathrm{deg} / \mathrm{sec}$, because the extent of perceived motion smear was longer than in the 8 and $4 \mathrm{deg} / \mathrm{sec}$ conditions (see Figure 3B, triangles).

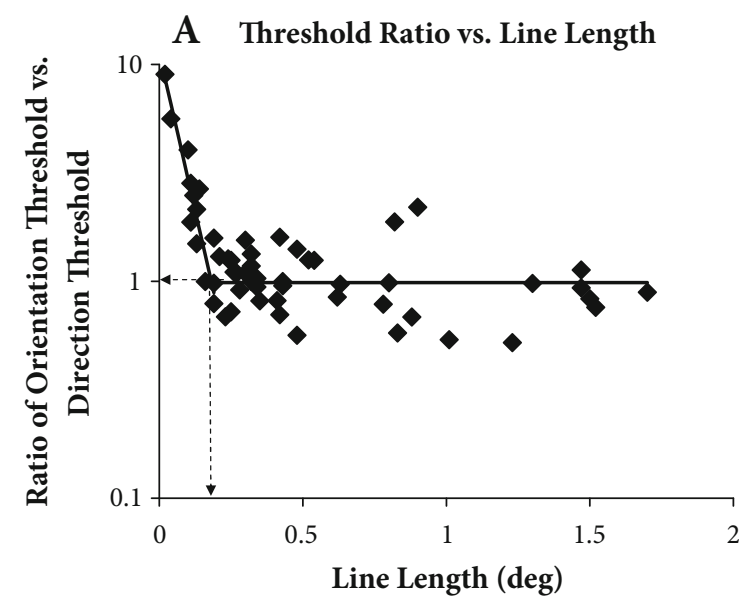

B Direction Threshold vs. Orientation Threshold

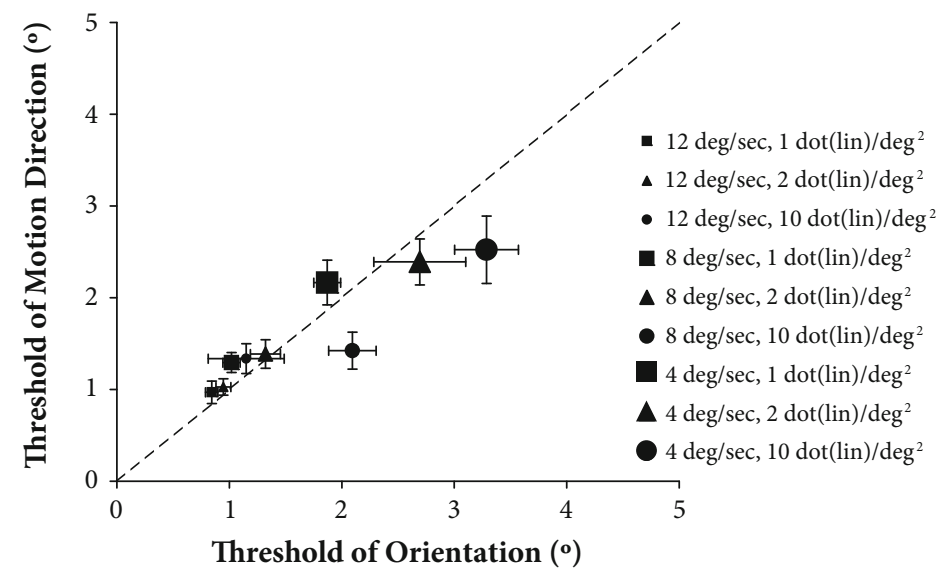

Figure 5. (A) The ratio between the thresholds for orientation discrimination and direction-of-motion discrimination is plotted against the matched values of line length and perceived motion smear, for each combination of subject and experimental condition. The data are fit by two straight lines, the second of which was constrained to have a slope of zero. The $x$-axis value that corresponds to the intersection between the two lines $(0.18 \mathrm{deg})$ indicates the length below which the thresholds for orientation are higher than those for direction of motion. For lengths longer than $0.18 \mathrm{deg}$, the two thresholds are essentially identical. (B) The average direction-of-motion thresholds are plotted against the average line-orientation thresholds for corresponding conditions of dot or line density and smear or line length. Vertical and horizontal error bars are $\pm 1 S E$, across subjects. Thresholds for direction-of-motion and orientation discrimination are equal on the diagonal 1:1 line. Consistent with the data presented in panel A, the plotted points are closest to the 1:1 line for combinations of high RD velocity and low density. 


\section{DISCUSSION}

The purpose of the present study was to investigate whether the extent of perceived motion smear influences direction-of-motion discrimination. Many earlier authors had expressed the extent of perceived motion smear in terms of duration, by converting the length of perceived motion smear from units of angular length to time. This representation allows the inferred duration of visual persistence to be compared for stimuli that move at different speeds (Chen et al., 1995; Hogben \& Di Lollo, 1985). Previous investigations had found that the duration of perceived motion smear decreases as the density of stimulus elements increases. For example, Chen et al. (1995) reported that the extent of perceived motion smear is around $100 \mathrm{msec}$ for a single bright dot that moves at $10 \mathrm{deg} / \mathrm{sec}$ for $160 \mathrm{msec}$, and decreases to approximately $30 \mathrm{msec}$ when the dot density increases to $7.5 \mathrm{dots} / \mathrm{deg}^{2}$. The extent of perceived motion smear in the present investigation shows a very similar decrease with dot density, when expressed in terms of duration. In particular, the average duration of perceived motion smear decreases from approximately $90 \mathrm{msec}$ for a single dot that moves at $8 \mathrm{deg} / \mathrm{sec}$ for $200 \mathrm{msec}$, to $30 \mathrm{msec}$ when the dot density increases to $10 \mathrm{dots} / \mathrm{deg}^{2}$. Because the purpose of the present study was to compare direction-of-motion discrimination with orientation discrimination, it was more appropriate to express the extent of perceived motion smear in units of visual angle, rather than time.

It is noteworthy that direction-of-motion discrimination worsens as the RD density increases, despite the opportunity for a greater summation of motion signals. This aspect of our results is consistent with most of the previous psychophysical studies that have compared direction discrimination for single dots and moving RD patterns. For example, the average threshold reported by Westheimer and Wehrhahn (1994) for direction-of-motion discrimination with RD stimuli was $1.55^{\circ}$, which was slightly but significantly higher than the direction-of-motion threshold of $1.43^{\circ}$ for a single moving dot. Similar results were also reported by De Bruyn and Orban (1988) and Matthews and Welch (1997), although not by Matthews and Allen (2005). Because the single-dot stimuli used by Matthews and Allen appeared at random locations within a 3-deg spatial window, poorer direction discrimination for this stimulus than for their denser RD targets may reflect a decrease in performance for off-foveal retinal locations.

The reason that we offer for superior direction-of-motion discrimination when using sparse as opposed to dense RD targets is that the angular extent of perceived motion smear decreases as the density of RDs in the moving stimulus increases. If the length of perceived motion smear were comparable in the high-density and single-dot conditions, then direction-of-motion thresholds would be expected to be more similar. Indeed, one of our subjects reported substantially more motion smear for a $10 \mathrm{dots} / \mathrm{deg}^{2}$ target moving at $12 \mathrm{deg} / \mathrm{sec}$ than did the other subjects in our study. For this subject, the difference between the direction-of-motion thresholds at $12 \mathrm{deg} / \mathrm{sec}$ for high and low RD densities was correspondingly smaller than for the other subjects. This observed negative relationship between RD density and direction-of-motion discrimination is consistent with our interpretation that direction-of-motion discrimination is facilitated by the presence of perceived motion smear.

The results of some earlier investigations are consistent with this interpretation. In their study of direction-ofmotion discrimination, Burr and Ross (2002) presented 200 RDs per frame in an area of $300 \mathrm{deg}^{2}$, which corresponds to a density of $0.67 \mathrm{dot} / \mathrm{deg}^{2}$. Although the lifetime of the moving RDs was limited, the low density and the high speed of their moving RD stimuli should have allowed the observers to perceive rudimentary "streak" information. Burr and Ross found that direction-of-motion discrimination is most impaired by visual noise that is oriented parallel to the direction of motion, which would be expected to maximally reduce the visibility of the streak information. Earlier, Geisler (1999) determined that an oriented adapting stimulus distorts the perceived direction of motion for an isolated suprathreshold dot that moves at $10 \mathrm{deg} / \mathrm{sec}$, and has little or no effect if the velocity of the dot is $2.5 \mathrm{deg} / \mathrm{sec}$. On the basis of our results, a bright dot that moves on a dark field would be expected to generate approximately $90 \mathrm{msec}$ of visible persistence, corresponding to visible motion smear of 0.9 and $0.23 \mathrm{deg}$ for dot velocities of 10 and $2.5 \mathrm{deg} / \mathrm{sec}$, respectively. Because the diameter of the dot in Geisler's experiment was $0.2 \mathrm{deg}$, it is reasonable to assume that only the longer extent of perceived smear, produced when the dot velocity was $10 \mathrm{deg} / \mathrm{sec}$, would provide useful information about the direction of motion.

However, in another experiment, Geisler (1999) showed that the orientation of a masking stimulus affects the detection of a moving target at a velocity as low as $1 \mathrm{deg} / \mathrm{sec}$, which is probably too slow to generate perceived motion smear. Geisler concluded that it is not necessary to perceive motion streaks in order for them to aid in the detection of motion. Burr and Ross (2002), on the other hand, found no evidence that motion streaks affected the detection of their moving RD target. They attributed the difference between their results and Geisler's to the more highly sampled motion stimuli in their study. Our experiments do not address the relationship between the detection of motion and the extent of perceived motion smear. It is possible that the perception of motion streaks improves direction-of-motion discrimination, but that visibility is not required to facilitate the detection of a moving target.

Some previous studies have reported that the discrimination of line orientation is almost twice as sensitive as the discrimination of motion direction (Matthews \& Welch, 1997; Westheimer \& Wehrhahn, 1994). In contrast, we found no significant difference $(p>.90)$ between direction-of-motion and orientation thresholds, when the extent of perceived smear and/or line length was longer than approximately $0.2 \mathrm{deg}$. One possible reason for this discrepancy is that previous studies compared directionof-motion and orientation thresholds using line lengths equated to the entire trajectory of motion, rather than to the extent of perceived motion smear (Matthews \& Welch, 1997; Westheimer \& Wehrhahn, 1994). For stimulus du- 
rations greater than approximately $50 \mathrm{msec}$, the extent of perceived motion smear is typically less than the trajectory of the stimulus motion on the retina (Burr, 1980; Chen et al., 1995). Because orientation discrimination improves as line length increases, orientation-discrimination thresholds would be expected to be better than direction discrimination when the length of the orientation stimulus is set equal to the length of the entire motion trajectory.

It is also possible that fixation instability exerts different effects on the discrimination of motion direction and stimulus orientation. For example, fixational drifts and saccades that occur during the presentation of a moving stimulus alter the trajectory of the motion on the retina, leading to errors in the perceived direction of motion (Souman, Hooge, \& Wertheim, 2005). In contrast, the horizontal and vertical components of fixational eye movements change the location, but not the orientation of parallel line stimuli on the retina. Although the retinal-image orientation of the line stimuli would be altered by torsional eye movements, the standard deviation of eye torsion during fixation averages less than $0.3^{\circ}$ (Ferman, Collewijn, Jansen, \& van den Berg, 1987; Morisita \& Yagi, 2001; Ott, Seidman, \& Leigh, 1992; van Rijn, van der Steen, \& Collewijn, 1994), which is considerably smaller than the thresholds for normal orientation discrimination (Ukwade et al., 2002). A noteworthy aspect of our experimental design was that it provided a fixation stimulus except during the $200-\mathrm{msec}$ duration of the moving or oriented stimuli, which allowed our subjects to maintain relatively good fixation while eliminating visible references.

However, fixation instability may have played a role in the results obtained by Matthews and Allen (2005), which led them to conclude that direction-of-motion discrimination is mediated by direction-selective rather than orientation-based mechanisms. Matthews and Allen asked their subjects to judge the direction difference between a standard and a sequentially presented test RD stimulus, with either the same or opposite directions of motion. The moving stimuli were assumed to produce the same orientation information in both conditions. However, eye drift can introduce an additional component of retinal-image motion, which is similar for stimuli that move in the same direction and dissimilar for stimuli that move in opposite directions. For example, during target motion to the right, upward eye drift introduces a component of motion that is consistent with a downward target trajectory. During target motion to the left, the same upward eye drift produces a motion component consistent with an upward target trajectory. Because of the added retinal-image motion that results from eye drifts, orientation information from the retinal trajectory of motion will vary more for stimuli that move in opposite directions than for those that move in the same direction, which could account for the reported differences in direction-discrimination thresholds. Another relevant aspect of the stimuli used by Matthews and Allen is that, in most of their experiments, the density of the RD pattern was 4.2 dots/ $\mathrm{deg}^{2}$ and the velocity of RD motion was $8 \mathrm{deg} / \mathrm{sec}$. These values of density and velocity would be expected to produce a substantial reduction of perceived motion smear, in comparison with that gener- ated by an isolated moving target (see, e.g., Chen et al., 1995). In the absence of perceived motion smear, the subjects in the study by Matthews and Allen would have been forced to rely primarily on motion signals to mediate their direction judgments.

Our results indicate that the thresholds to discriminate direction of motion and orientation are indistinguishable when the extent of perceived smear or line length is greater than approximately $0.2 \mathrm{deg}$, whereas directionof-motion discrimination is better than the discrimination of line orientation when the extent of perceived smear or line length is shorter. The highly similar results for discriminating the direction of $\mathrm{RD}$ motion and the orientation of flashed stationary lines for lengths greater than 0.2 deg suggest that both types of discrimination are mediated primarily by neural mechanisms that rely on orientation information. On the other hand, when the extent of perceived motion smear is less than $0.2 \mathrm{deg}$, the visual system presumably uses the more sensitive motion signals instead of orientation information to mediate direction discrimination. When the extent of perceived motion smear is close to $0.2 \mathrm{deg}$, it is possible that direction-ofmotion information depends on an integration of motion and orientation signals, weighted according to the relative precision of each source of information (Ernst \& Banks, 2002; Howard, 1997; Young, Landy, \& Maloney, 1993).

However, as suggested above in our discussion of Geisler's (1999) results, the length of perceived motion smear at which direction-of-motion discrimination is indistinguishable from orientation discrimination probably depends on the dimensions of the moving target. For example, whereas $0.2 \mathrm{deg}$ of perceived motion smear can provide useful information about orientation for the small moving targets in this study, this same extent of perceived smear would not be expected to give precise information about orientation if the diameter of the moving targets were larger. For moving targets that consist of larger elements, we speculate that the threshold for direction-of-motion discrimination would become equal to the threshold for orientation discrimination only when the extent of perceived motion smear is considerably longer than $0.2 \mathrm{deg}$.

\section{AUTHOR NOTE}

We thank Saumil Patel for useful comments and Susana Chung for help with curve fitting. This study was supported by Research Award R01 EY05068 and Core Center Grant P30 EY07551 from the National Eye Institute and Research Award 003652-0185-2001 from the Texas Advanced Research Program. Correspondence concerning this article should be addressed to H. E. Bedell, College of Optometry, 505 J. Davis Armistead Building, University of Houston, Houston, TX 77204-2020 (e-mail: hbedell@optometry.uh.edu).

\section{REFERENCES}

Adelson, E. H., \& Movshon, J. A. (1982). Phenomenal coherence of moving visual patterns. Nature, 300, 523-525.

Azzopardi, P., \& Cowey, A. (2001). Motion discrimination in cortically blind patients. Brain, 124, 30-46.

Barlow, H. B., \& Olshausen, B. A. (2004). Convergent evidence for the visual analysis of optic flow through anisotropic attenuation of high spatial frequencies. Journal of Vision, 4, 415-426.

Bidwell, S. (1899). Curiosities of light and sight. London: Swan Sonnenschein. 
Blanke, O., Landis, T., Mermoud, C., Spinelli, L., \& Safran, A. B. (2003). Direction-selective motion blindness after unilateral posterior brain damage. European Journal of Neuroscience, 18, 709-722.

Bowen, R. W., Pola, J., \& Matin, L. (1974). Visual persistence: Effects of flash luminance, duration and energy. Vision Research, 14, 295-303.

Britten, K. H., Shadlen, M. N., Newsome, W. T., \& Movshon, J. A. (1992). The analysis of visual motion: A comparison of neuronal and psychophysical performance. Journal of Neuroscience, 12, 4745-4765.

BURR, D. C. (1980). Motion smear. Nature, 284, 164-165.

Burr, D. C., \& Ross, J. (2002). Direct evidence that "speedlines" influence motion mechanisms. Journal of Neuroscience, 22, 8661-8664.

Chen, S., Bedell, H. E., \& OGMEN, H. (1995). A target in real motion appears blurred in the absence of other proximal moving targets. $\mathrm{Vi}$ sion Research, 35, 2315-2328.

Cheng, K., Fujita, H., Kanno, I., Miura, S., \& Tanaka, K. (1995). Human cortical regions activated by wide-field visual motion: An $\mathrm{H}_{2}{ }^{15} \mathrm{O}$ PET study. Journal of Neurophysiology, 74, 413-427.

Coltheart, M. (1980). Iconic memory and visible persistence. Perception \& Psychophysics, 27, 183-228.

Cornette, L., Dupont, P., Rosier, A., Sunaert, S., Van Hecke, P., Michiels, J., ET AL. (1998). Human brain regions involved in direction discrimination. Journal of Neurophysiology, 79, 2749-2765.

De Bruyn, B., \& OrBAN, G. A. (1988). Human velocity and direction discrimination measured with random dot patterns. Vision Research, 28, 1323-1335.

ERTST, M. O., \& BanKs, M. S. (2002). Humans integrate visual and haptic information in a statistically optimal fashion. Nature, $\mathbf{4 1 5}$, 429-433.

Ferman, L., Collewijn, H., Jansen, T. C., \& van den Berg, A. V. (1987). Human gaze stability in the horizontal, vertical and torsional directions during voluntary head movements, evaluated with a threedimensional scleral induction coil technique. Vision Research, 27, 811-828.

GEISLER, W. S. (1999). Motion streaks provide a spatial code for motion direction. Nature, 400, 65-69.

Geisler, W. S., Albrecht, D. G., Crane, A. M., \& Stern, L. (2001). Motion direction signals in the primary visual cortex of cat and monkey. Visual Neuroscience, 18, 501-516.

Hogben, J. H., \& Di Lollo, V. (1985). Suppression of visible persistence in apparent motion. Perception \& Psychophysics, 38, 450-460.

Holtzman, J. D., Sedgwick, H. A., \& Festinger, L. (1978). Interaction of perceptually monitored and unmonitored efferent commands for smooth pursuit eye movements. Vision Research, 18, 1545-1555.

HowARD, I. P. (1997). Interactions within and between the spatial senses. Journal of Vestibular Research, 7, 311-345.

HUBEL, D. H., \& WIESEL, T. N. (1968). Receptive fields and functional architecture of monkey striate cortex. Journal of Physiology, 195, 215-243.

KoSNIK, W., FIKRE, J., \& SEKULER, R. (1985). Improvement in direction discrimination: No role for eye movements. Perception \& Psychophysics, 38, 554-558.

MARR, D., \& Ullman, S. (1981). Directional selectivity and its use in early visual processing. Proceedings of the Royal Society of London: Series $B, 211,151-180$.

MATtheWS, N., \& AlleN J. (2005). The role of speed lines in subtle direction judgments. Vision Research, 45, 1629-1640.
Matthews, N., \& Welch, L. (1997). Velocity-dependent improvements in single-dot direction discrimination. Perception \& Psychophysics, 59, 60-72.

MCDOUGALL, W. (1904). The sensations excited by a single momentary stimulation of the eye. British Journal of Psychology, 1, 78-113.

Morisita, M., \& YAGI, T. (2001). The stability of human eye orientation during visual fixation and imagined fixation in three dimensions. Auris Nasus Larynx, 28, 301-304.

OTT, D., SEIDMAN, S. H., \& LEIGH, R. J. (1992). The stability of human eye orientation during visual fixation. Neuroscience Letters, 142, 183-186.

Pack, C. C., Livingstone, M. S., Duffy, K. R., \& Born, R. T. (2003). End-stopping and the aperture problem: Two-dimensional motion signals in macaque V1. Neuron, 39, 671-680.

Peterson, M. R., Li, B., \& Freeman, R. D. (2004). The derivation of direction selectivity in the striate cortex. Journal of Neuroscience, 24, 3583-3591.

Purushothaman, G., \& Bradley, D. C. (2005). Neural population code for fine perceptual decisions in area MT. Nature Neuroscience, 8, 99-106.

Purushothaman, G., Ögmen, H., Chen, S., \& Bedell, H. E. (1998). Motion deblurring in a neural network model of retino-cortical dynamics. Vision Research, 38, 1827-1842.

Salzman, C. D., Britten, K. H., \& Newsome, W. T. (1990). Cortical microstimulation influences perceptual judgements of motion direction. Nature, 346, 174-177.

Singh, K. D., Smith, A. T., \& Greenlee, M. W. (2000). Spatiotemporal frequency and direction sensitivities of human visual areas measured using fMRI. NeuroImage, 12, 550-564.

Souman, J. L., HoOge, I. T. C., \& WerTheim, A. H. (2005). Vertical object motion during horizontal ocular pursuit: Compensation for eye movements increases with presentation duration. Vision Research, 45, 845-853.

ToNG, J., PATEL, S. S., \& Bedell, H. E. (2005). Asymmetry of perceived motion smear during head and eye movements: Evidence for a dichotomous neural categorization of retinal image motion. Vision Research, 45, 1519-1524.

UKWADE, M. T., BEDELL, H. E., \& White, J. M. (2002). Orientation discrimination and variability of torsional eye position in congenital nystagmus. Vision Research, 42, 2395-2407.

VAN RIJN, L. J., VAN DER STEEN, J., \& COLLEWIJN, H. (1994). Instability of ocular torsion during fixation: Cyclovergence is more stable than cycloversion. Vision Research, 34, 1077-1087.

Westheimer, G., \& Wehrhahn, C. (1994). Discrimination of direction of motion in human vision. Journal of Neurophysiology, 71, 33-37.

Williams, D. W., \& Sekuler, R. (1984). Coherent global motion percepts from stochastic local motions. Vision Research, 24, 55-62.

Williams, R. A., ENOCH, J. M., \& Essock, E. A. (1984). The resistance of selected hyperacuity configurations to retinal image degradation. Investigative Ophthalmology \& Visual Science, 25, 389-399.

YoUNG, M. J., LANDY, M. S., \& MALONEY, L. T. (1993). A perturbation analysis of depth perception from combinations of texture and motion cues. Vision Research, 33, 2685-2696.

Zohary, E., SCASE, M. O., \& BRADDICK, O. J. (1996). Integration across directions in dynamic random dot displays: Vector summation or winner take all? Vision Research, 36, 2321-2331.

(Manuscript received September 30, 2005; revision accepted for publication March 7, 2006.) 